\title{
Generic optimality conditions for semi-algebraic convex programs
}

\author{
J. Bolte $^{*} \quad$ A. Daniilidis ${ }^{\dagger} \quad$ A.S. Lewis ${ }^{\ddagger}$
}

December 20, 2010

\begin{abstract}
We consider linear optimization over a nonempty convex semialgebraic feasible region $F$. Semidefinite programming is an example. If $F$ is compact, then for almost every linear objective there is a unique optimal solution, lying on a unique "active" manifold, around which $F$ is "partly smooth", and the second-order sufficient conditions hold. Perturbing the objective results in smooth variation of the optimal solution. The active manifold consists, locally, of these perturbed optimal solutions; it is independent of the representation of $F$, and is eventually identified by a variety of iterative algorithms such as proximal and projected gradient schemes. These results extend to unbounded sets $F$.
\end{abstract}

Key words: convex optimization, sensitivity analysis, partial smoothness, identifiable surface, active sets, generic, second-order sufficient conditions, semi-algebraic.

AMS Subject Classification Primary 90C31 ; Secondary 14P10, 32B20, 49K40, 90C05

*TSE (GREMAQ, Université Toulouse 1), 21 alle de Brienne, 31000 Toulouse, France. Research supported in part by grant ANR-08-BLAN-0294-01. www.ecp6.jussieu.fr/pageperso/bolte

${ }^{\dagger}$ Matemàtiques, Universitat Autònoma de Barcelona, Spain. Research supported in part by grants ANR-05-BLAN-0248-01 (France) and MTM2008-06695-C03-03 (Spain). mat.uab.es/ ${ }^{\sim}$ arisd

‡ORIE, Cornell University, Ithaca, NY 14853, U.S.A. Research supported in part by NSF Grant DMS-0806057. people.orie.cornell.edu/ ${ }^{\sim}$ aslewis 


\section{Introduction}

Optimizers approach problems from diverse perspectives, designing algorithms and analyzing their convergence behavior, studying sensitivity analysis with respect to data perturbation, and, relatedly, investigating duality theory and shadow prices. In most cases, whether in theory or computational practice, we make assumptions about prevailing optimality conditions, a canonical example being the second-order sufficient conditions for classical nonlinear programming [28].

That such optimality conditions typically hold can be justified rigorously via Sard's Theorem. To take the most basic example, when considering smooth equality constraints $F(x)=b$, we usually assume that the Jacobian of the map $F$ has full rank at some feasible solution of interest. Providing $F$ is sufficiently smooth, Sard's Theorem guarantees that for a generic vector $b$, this assumption holds at any feasible solution. "Generic" in this context means that the assertion holds for almost all $b$, in the sense of Lebesgue measure, and hence, for example, almost surely for a random $b$ having an everywhere strictly positive probability density function.

A classic paper of Spingarn and Rockafellar [40] explains how such arguments show the generic nature of the second-order sufficient conditions: see also [39]. The idea of studying optimization from a generic perspective dates back further, at least to Saigal and Simon's 1973 study [36] of the complementarity problem, and has persisted: see for example the studies of generic strict complementarity and primal and dual nondegeneracy for semidefinite programming by Alizadeh, Haeberly and Overton [1] and Shapiro [37], and for general conic convex programs by Pataki and Tunçel [29].

An important consequence of the classical second-order conditions is the existence of an "active manifold", consisting of those feasible points satisfying all the active constraints with equality. Perturbations to the objective function result in smooth perturbation of the optimal solution on this manifold. Classical active set algorithms attempt to find this manifold, thereby reducing the optimization problem to a much easier equality-constrained problem.

For convex programs in particular, a variety of algorithms, such as proximal and projected-gradient schemes, "identify" the active manifold automatically: after finite time, iterates generated by the algorithm must lie on the manifold. For example, Rockafellar [33] observed that the classical proximal point method converges finitely on a polyhedral function; the same holds for functions with the "weak sharp minimum" property introduced by Ferris [12]. 
Burke and Moré present an early survey on identification in the polyhedral case in [6].

Modern optimization considers a variety of models beyond the framework of classical nonlinear programming. Semidefinite programming is one example. This generality introduces more complexity into the active set methodology. For example, sensitivity analysis when a constraint involves a positive semidefinite matrix variable must consider not simply whether or not the variable is zero, but rather its rank: see for example [4]. Stability constraints on nonsymmetric matrices involve analogous subtleties [5].

In these more general frameworks, active set ideas can perhaps be better understood directly in terms of the geometry of the feasible region rather than its defining constraints, adopting an intrinsic approach rather than a structural one. This idea motivates the definition of a weak sharp minimum [12], and the generalization to "identifiable surfaces" in convex optimization due to Wright [43]. The equivalent idea of an active manifold with respect to which the feasible region is "partly smooth" was introduced by Lewis in [21]; like the weak sharp minimum idea, this approach is intrinsically geometric (and furthermore extends to the nonconvex case). A closely related idea, " $\mathcal{U V}$-decompositions" of convex functions, was developed by Lemaréchal, Oustry and Sagastizábal [20, 19].

Just as in classical cases, partial smoothness guarantees that a wide variety of iterative algorithms necessarily arrive on the active manifold after finite time [43, 13], raising the hope of accelerating the algorithms using second-order information. This hope motivated the $\mathcal{U V}$-decomposition idea, and was pursued further by Mifflin and Sagastizábal [25, 26] and recently by Daniilidis, Sagastizábal and Solodov [9]. A general approach, based on a proximal algorithm for composite optimization, is sketched by Lewis and Wright in [22], and broad techniques for estimating identifiable surfaces computationally are discussed in [23].

Partial smoothness is a relatively strong assumption: it does not, for example, subsume the subtleties of sensitivity analysis investigated extensively by Bonnans and Shapiro in [4], and it evades the technical complexities of second-order nonsmooth analysis, as described in Rockafellar and Wets' monograph [34]. Nonetheless, [21] argues that partial smoothness is a broad, intuitive, and powerful conceptual tool, and that a corresponding analogue of the second-order sufficient conditions suffices for a thorough and substantial generalization of classical nonlinear programming sensitivity analysis. In further analogy to the classical case, we would naturally expect algorithmic 
convergence analysis to rely on the same assumption.

In this work, we follow Spingarn and Rockafellar's philosophy [40] in asking whether an assumption of partial smoothness and second-order sufficiency is usually justified. We prove, for a large class of linear optimization problems over convex feasible regions, that this assumption indeed holds generically.

As remarked in [40], the key ingredients to any such argument about generic behavior is the class of problems under consideration and the precise notion of "generic" we use. The results of [40] fixed an objective and constraint functions, allowed linear perturbations to the objective and constant perturbations to the constraints, and proved a measure-theoretic result about the second-order conditions via Sard's Theorem. Both [1] and [37] use rather analogous arguments to prove that strict complementarity and primal and dual nondegeneracy are generic properties of semidefinite programs; using a very different technique based on the boundary behavior of convex sets, Pataki and Tunçel [29] generalized these results to general conic convex programs. Ioffe and Lucchetti [16] adopt a more abstract, topological approach, allowing very general perturbations to the optimization problem but proving a result instead about "well-posedness" [10]. Our approach, while notable for the generality of the feasible regions considered, is more concrete, combining some of the spirit of [40] and [29]: we aim to understand the generic nature of second-order sufficient conditions, like [40], but we make no assumption about how the feasible region is presented; instead, we assume only that it is semi-algebraic. In a recent survey [15], Ioffe describes how a semi-algebraic version of Sard's Theorem applies to an analogue of Spingarn and Rockafellar's result on generic optimality conditions. (Pataki and Tunçel attribute a semi-algebraic version of their generic strict complementarity and nondegeneracy result to Renegar.)

A set defined by finitely-many polynomial inequalities is called basic semialgebraic; any set that can be represented as a finite union of such sets is simply called semi-algebraic. Semi-algebraic sets comprise a rich class that is stable under many mathematical operations. They are often easy to recognize, even without an explicit representation as a union of basic sets, as a consequence of the Tarski-Seidenberg Theorem, which states, loosely, that the projection of a semi-algebraic set is semi-algebraic. For example, the feasible region of any semidefinite program is semi-algebraic. A good resource on semi-algebraic geometry is [2].

As our main result, we prove that, given any fixed nonempty closed convex semi-algebraic set, corresponding to a generic linear objective function is a 
unique optimal solution, lying on a unique active manifold, and for which the partly smooth second-order sufficient conditions hold. The active manifold is independent of any particular representation of the semi-algebraic set as a union of basic sets, and the optimal solution varies smoothly on it (in fact giving a complete local description of it) under local perturbations to the objective function. In particular, this result holds for any semidefinite program.

The term "generic" for a large subset of Euclidean space has been used in a variety of mathematical senses. Spingarn and Rockafellar [40] mean a full-measure subset - its complement has Lebesgue measure zero-whereas topologically generic means that the subset contains a countable intersection of dense open subsets. These two notions are incompatible in general, but fortunately, as we explain in Section 3, the distinction collapses and the idea dramatically simplifies for a semi-algebraic sets, because such sets "stratify" into finite unions of smooth manifolds. For semi-algebraic sets, therefore, we have three equivalent properties: full-measure, topologically generic, and dense.

Previous work on generic optimality conditions has been mostly structural, focusing on some given functional presentation of the feasible region, rather than on its intrinsic geometry. Spingarn and Rockafellar's work [40] concerns classical smooth constraint systems, and Ioffe's semi-algebraic version [15] is analogous. Alizadeh, Haeberly and Overton [1] derive results for linear semidefinite programs, extended to general conic convex systems by Pataki and Tunçel [29]; Shapiro and Fan [38] and Shapiro [37] focus on nonlinear semidefinite programs. Such structural approaches reflect the presentation of optimization problems in practice, and are very general: in particular, [3] is a powerful toolkit for semidefinite representation of convex sets (that is, as affine pre-images of the semidefinite cone).

For comparison purposes, the approach to second-order conditions developed by Bonnans and Shapiro in [4] is particularly general and instructive. They consider smooth pre-images of general closed convex cones, and, like $[38,37,1]$, present in [4, Section 4.6.1] an appropriate generalization of the classical full rank condition. This transversality condition - called constraint nondegeneracy by Robinson [31], and also discussed in detail in the context of nonlinear semidefinite programming by Sun in [41], is generic, as a consequence of Sard's theorem, providing the problem parametrization is sufficiently rich [4, Section 5.3.1]. Another important ingredient of second-order analysis, the quadratic decay condition, is also generic in semidefinite pro- 
gramming, since it is equivalent (see [4, Theorem 5.91]) to uniqueness of the optimal solution along with a suitable analogue of the classical "strict complementarity" condition, known to be generic [1, 29]. The active manifold also emerges naturally using this approach, via a standard application of the transversality condition, assuming a powerful property of the underlying cone called "cone reducibility" [4, Definition 3.135]. While cone-reducibility is in general nontrivial to verify, some careful calculations show that the semidefinite cone in particular is cone reducible [4, Example 3.140], and furthermore products of cone reducible cones are cone reducible.

Despite the great generality of these concrete, structural approaches, an intrinsic theory, based on the feasible region itself rather than functional descriptions thereof, has a certain appeal. We make no assumption whatsoever about the presentation of feasible region assuming only that it is semialgebraic. In other respects, our assumptions are quite restrictive: we deal only with the convex case, and only consider perturbations to the objective, taking what is possibly just a first step towards a more general theory. Even the theoretical gain in generality in considering semi-algebraic sets is unclear, since, despite considerable interest and effort, an example of a semi-algebraic convex set that is not semidefinite representable remains undiscovered [14]. Nonetheless, this semi-algebraic approach is interesting: the main result is independent of the choice of presentation of the feasible region (a choice that may influence the corresponding genericity result in a complex fashion), the proof technique is novel in this context, the generic conclusion is stronger and more concrete (holding on a set that is dense and open rather than just full measure), and the sole assumption of semi-algebraicity is typically immediate to verify, due to the Tarski-Seidenberg Theorem.

The stratification property on which our theoretical development fundamentally depends is not confined to the class of semi-algebraic sets. It holds more generally for "subanalytic" sets, and indeed for any "tame" class of sets: see for example [8] for a short introduction to tame geometry. Consequently, while all results are stated for semi-algebraic sets, the authors believe analogous results hold for tame sets, and furthermore, that tame-geometric techniques show great promise in optimization theory more generally (see for example Ioffe's survey [15]). However, to lighten the exposition, we do not pursue this extension here.

Our approach in this paper relies heavily on convexity. However, many of the basic ideas driving this development extend to nonconvex settings, a central example being the normal cone and its continuity properties. Nonconvex 
variational analysis has grown into a complete, powerful and elegant theory over the past several decades: fine expositions may be found in [27, 7, 34]. Extending the results described in this paper to nonconvex settings is the topic of ongoing research.

Our exposition blends three relatively unfamiliar techniques for an optimization audience: the notion of a generic problem instance, semi-algebraic geometry, and partial smoothness. We strive, however, for a self-contained approach, introducing and discussing the key concepts as we need them, and assuming nothing beyond classical convex analysis.

\section{Preliminary results}

We begin, in this section, with some routine convex analysis, following the notation of [32], unless otherwise stated. Throughout this work we deal with the Euclidean space $\mathbf{R}^{n}$ equipped with the usual scalar product $\langle\cdot, \cdot\rangle$ and the corresponding Euclidean norm $|\cdot|$. We denote by $B(x, r)$ the closed ball with center $x \in \mathbf{R}^{n}$ and radius $r>0$. A subset of $\mathbf{R}^{n}$ is a cone if it contains zero and is closed under nonnegative scalar multiplication. We denote by $S^{n-1}$ the unit sphere of $\mathbf{R}^{n}$. Given any set $E \subset \mathbf{R}^{n}$, we denote by $\bar{E}$ its closure.

Notation. Throughout this paper, unless otherwise stated, we consider a fixed nonempty compact convex set $F \subset \mathbf{R}^{n}$, and study the set of maximizers of the linear optimization problem

$$
\max _{F}\langle c, \cdot\rangle
$$

for vectors $c \in \mathbf{R}^{n}$. The linear case is in some sense not restrictive, because a nonlinear optimization problem $\max _{F} f$ could be rephrased as the linear problem $\max \{t: t \leq f(x), x \in F, t \in \mathbf{R}\}$.

A point $\bar{x} \in F$ is a maximizer if and only if $c$ lies in the normal cone

$$
N_{F}(\bar{x})=\left\{c \in \mathbf{R}^{n}:\langle c, x-\bar{x}\rangle \leq 0 \text { for all } x \in F\right\} .
$$

We call a maximizer $\bar{x}$ nondegenerate if in fact $c$ lies in the relative interior of the normal cone: $c \in \operatorname{ri} N_{C}(\bar{x})$.

For an arbitrary convex set $F \subset \mathbf{R}^{n}$ (possibly unbounded), we say that the objective function $\langle c, \cdot\rangle$ decays quadratically on $F$ around a maximizer $\bar{x}$ if there exists a constant $\delta^{\prime}>0$ such that

$$
\langle c, \bar{x}\rangle \geq\langle c, x\rangle+\delta^{\prime}|x-\bar{x}|^{2} \quad \text { for all } x \in F \cap B\left(\bar{x}, \delta^{\prime}\right) ;
$$


see for example [4]. Although this is a local condition, it implies uniqueness of the maximizer: $\langle c, \bar{x}\rangle>\langle c, x\rangle$ whenever $\bar{x} \neq x \in B\left(\bar{x}, \delta^{\prime}\right)$, and hence whenever $\bar{x} \neq x$, by convexity. On the other hand, quadratic decay can easily fail, even around a unique nondegenerate maximizer. For example, the point zero is the unique maximizer for the problem $\max \left\{-x_{2}: x_{2} \geq\left|x_{1}\right|^{\frac{3}{2}}\right\}$, and it is nondegenerate, but quadratic decay fails.

If the set $F$ is compact, quadratic decay is in fact a global condition: it simplifies to the existence of a constant $\delta>0$ such that

$$
\langle c, \bar{x}\rangle \geq\langle c, x\rangle+\delta|x-\bar{x}|^{2} \quad \text { for all } x \in F .
$$

Indeed, if property (1) holds, then uniqueness of the maximizer $\bar{x}$ implies that the continuous function

$$
x \mapsto \frac{\langle c, \bar{x}-x\rangle}{|x-\bar{x}|^{2}}
$$

is strictly positive on the compact set $\left\{x \in F:|x-\bar{x}| \geq \delta^{\prime}\right\}$; if we denote the minimum value of this function by $\delta^{\prime \prime}>0$, then property (2) holds with $\delta=\min \left\{\delta^{\prime}, \delta^{\prime \prime}\right\}$.

The set of maximizers $\operatorname{argmax}_{F}\langle c, \cdot\rangle$ is called the exposed face of the set $F$ corresponding to the vector $c$. In particular, the set $F$ is itself an exposed face (corresponding to $c=0$ ): all other exposed faces we call proper. The optimal value $\max _{F}\langle c, \cdot\rangle$, as a function of $c$, is called the support function, denoted $\sigma_{F}$ : under our standing assumption that $F$ is compact, the support function is a continuous and positively homogeneous convex function. Via standard convex analysis [32], we know that $\operatorname{argmax}_{F}\langle c, \cdot\rangle$ is the nonempty compact convex set $\partial \sigma_{F}(c)$, where $\partial$ denotes the convex subdifferential. We denote by $x_{c}$ the optimal solution of minimum norm:

$$
x_{c}=\operatorname{argmin}\left\{|x|: x \in \operatorname{argmax}_{F}\langle c, \cdot\rangle\right\} .
$$

Notice the homogeneity property

$$
x_{\lambda c}=x_{c} \quad \text { for all } c \in \mathbf{R}^{n} \text { and } \lambda>0 \text {. }
$$

Clearly $\langle c, \cdot\rangle$ decays quadratically around a maximizer if and only if there exists a constant $\delta_{c}>0$ such that

$$
\left\langle c, x_{c}\right\rangle \geq\langle c, x\rangle+\delta_{c}\left|x-x_{c}\right|^{2} \quad \text { for all } x \in F \text {. }
$$


We aim to show good behavior of the optimization problem $\max _{F}\langle c, \cdot\rangle$ for objective vectors $c$ lying in some large subset of $\mathbf{R}^{n}$, or equivalently, by scaling $c$, the sphere $S^{n-1}$.

We begin our development with a well-known result (cf. [11]) proved by an easy and standard argument.

Proposition 1 (Generic uniqueness). Consider a nonempty compact convex set $F \subset \mathbf{R}^{n}$. For all nonzero vectors $c$ lying in a topologically generic and full-measure cone in $\mathbf{R}^{n}$, the linear functional $\langle c, \cdot\rangle$ has a unique maximizer over $F$.

Proof. The set of optimal solutions is a singleton (namely $\left\{x_{c}\right\}$ ) if and only if the support function $\sigma_{F}$ is differentiable at $c$. Being a finite convex function, the set of points of differentiability is both topologically generic and full-measure in $\mathbf{R}^{n}$ [30], and by positive homogeneity, it is also closed under strictly positive scalar multiplication.

In fact a stronger result holds almost surely.

Proposition 2 (Generic quadratic decay). Consider a nonempty compact convex set $F \subset \mathbf{R}^{n}$. Denote by $K$ the set of vectors $c \in \mathbf{R}^{n}$ such that the linear functional $\langle c, \cdot\rangle$ decays quadratically around a maximizer over $F$. Then the cone $K \cup\{0\}$ is full-measure in $\mathbf{R}^{n}$.

Proof. It is easy to check that the set $K$ is closed under strictly positive scalar multiplication, so the set $K \cup\{0\}$ is certainly a cone. Alexandrov's Theorem ([34, Theorem 13.51]) applied to the finite convex function $\sigma_{F}$ shows that there exists a full-measure subset $A$ of $\mathbf{R}^{n}$ at each point of which $\sigma_{F}$ has a quadratic expansion. In particular, $\sigma_{F}$ has gradient $\nabla \sigma_{F}(c)=x_{c}$ for all vectors $c \in A$, and in view of [34, Definition 13.1(c)], we have, for any fixed vector $\bar{c} \in A$, that there exists a positive semidefinite matrix $S$ such that vectors $c \in \mathbf{R}^{n}$ near $\bar{c}$ satisfy

$$
\sigma_{F}(c)=\sigma_{F}(\bar{c})+\left\langle\nabla \sigma_{F}(\bar{c}), c-\bar{c}\right\rangle+\frac{1}{2}\langle S(c-\bar{c}), c-\bar{c}\rangle+o\left(|c-\bar{c}|^{2}\right) .
$$

Hence there exist constants $\varepsilon>0$ and $\rho>0$ such that for all $c \in B(\bar{c}, \varepsilon)$ we have

$$
\sigma_{F}(c) \leq \sigma_{F}(\bar{c})+\left\langle x_{\bar{c}}, c-\bar{c}\right\rangle+\frac{\rho}{2}|c-\bar{c}|^{2} .
$$


Further, we can clearly assume

$$
\varepsilon^{-1} \operatorname{diam}(F)<\rho .
$$

Now consider any point $x \in F$. Since the Fenchel conjugate of the function $\sigma_{F}$ is just the indicator function of $F$, we deduce successively

$$
\begin{aligned}
0=\sigma_{F}^{*}(x) & =\sup _{c \in \mathbf{R}^{n}}\left\{\langle x, c\rangle-\sigma_{F}(c)\right\} \\
& \geq \sup _{c \in B(\bar{c}, \varepsilon)}\left\{\langle x, c\rangle-\sigma_{F}(c)\right\} \\
& \geq \sup _{c \in B(\bar{c}, \varepsilon)}\left\{\langle x, c\rangle-\sigma_{F}(\bar{c})-\left\langle x_{\bar{c}}, c-\bar{c}\right\rangle-\frac{\rho}{2}|c-\bar{c}|^{2}\right\} \\
& =\sup _{c \in B(\bar{c}, \varepsilon)}\left\{\left\langle x-x_{\bar{c}}, c\right\rangle-\frac{\rho}{2}|c-\bar{c}|^{2}\right\} \\
& =\left\langle x-x_{\bar{c}}, \bar{c}\right\rangle+\sup _{u \in B(0, \varepsilon)}\left\{\left\langle x-x_{\bar{c}}, u\right\rangle-\frac{\rho}{2}|u|^{2}\right\} .
\end{aligned}
$$

In view of inequality (4), it is easy to see that the above supremum is attained at the point $u=\rho^{-1}\left(x-x_{\bar{c}}\right) \in B(0, \varepsilon)$. Replacing this value in the above inequality we deduce

$$
0 \geq\left\langle x-x_{\bar{c}}, \bar{c}\right\rangle+\frac{1}{2 \rho}\left|x-x_{\bar{c}}\right|^{2}, \quad \text { for all } x \in F .
$$

which yields the asserted equation with $\delta_{c}=(2 \rho)^{-1}$. Thus we have shown $A \subset K$, and the result follows.

We next follow an argument analogous to that of [29] to show that nondegeneracy is also a generic property. To prove this result, it suffices to consider the special case when the interior of the set $F$ contains the point zero. We then relate nondegeneracy to the facial structure of the polar set

$$
F^{\circ}=\left\{c \in \mathbf{R}^{n}:\langle c, x\rangle \leq 1 \text { for all } x \in F\right\},
$$

another compact convex set whose interior contains zero.

Proposition 3 (Normal representation of polar exposed faces). Suppose zero lies in the interior of the compact convex set $F \subset \mathbf{R}^{n}$. Then the proper exposed faces of the polar set $F^{\circ}$ are those sets of the form

$$
G_{\bar{x}}=\left\{c \in N_{F}(\bar{x}):\langle c, \bar{x}\rangle=1\right\},
$$


for points $\bar{x}$ on the boundary of $F$. Furthermore, any such exposed face has relative interior given by

$$
\operatorname{ri} G_{\bar{x}}=\left\{c \in \operatorname{ri} N_{F}(\bar{x}):\langle c, \bar{x}\rangle=1\right\} .
$$

Proof. For any point $\bar{x} \in F$ it is easy to see

$$
\left\{c \in N_{F}(\bar{x}):\langle c, \bar{x}\rangle=1\right\}=\left\{c \in F^{\circ}:\langle c, \bar{x}\rangle=1\right\}=\operatorname{argmax}_{F^{\circ}}\langle\cdot, \bar{x}\rangle .
$$

Thus any such set is certainly an exposed face, and if $\bar{x}$ is a boundary point of $F$ (and hence nonzero) then this exposed face must be proper, since it does not contain zero.

Conversely, by definition, any exposed face of $F^{\circ}$ has the form

$$
G=G_{z}=\left\{c \in F^{\circ}: \sigma_{F^{\circ}}(z)=\langle c, z\rangle\right\},
$$

for some vector $z \in \mathbf{R}^{n}$, and assuming $G$ is proper implies $z \neq 0$. By standard convex analysis, the support function $\sigma_{F^{\circ}}$ is identical to the gauge function $\gamma_{F}: \mathbf{R}^{n} \rightarrow \mathbf{R}_{+}$defined by

$$
\gamma_{F}(z)=\inf \left\{\lambda \in \mathbf{R}_{+}: z \in \lambda F\right\}
$$

Since $z \neq 0$, we know $\gamma_{F}(z)>0$, so we can define a point $\bar{x}=\gamma_{F}(z)^{-1} z$. By positive homogeneity, $\gamma_{F}(\bar{x})=1$, so $\bar{x}$ lies on the boundary of $F$, and

$$
G=\left\{c \in F^{\circ}: \gamma_{F}(z)=\langle c, z\rangle\right\} .
$$

The first part of the result follows.

To show the last equation, it suffices to prove that the sets

$$
\left\{c \in \mathbf{R}^{n}:\langle c, \bar{x}\rangle=1\right\} \text { and } \operatorname{ri} N_{F}(\bar{x})
$$

have nonempty intersection (see [32, Thm 6.5]). If not, there exists a separating hyperplane, and hence a nonzero vector $y \in \mathbf{R}^{n}$ and a number $\alpha \in \mathbf{R}$ satisfying, for all vectors $c \in \mathbf{R}^{n}$,

$$
\begin{aligned}
c \in N_{F}(\bar{x}) & \Rightarrow\langle c, y\rangle \leq \alpha \\
\langle c, \bar{x}\rangle=1 & \Rightarrow\langle c, y\rangle \geq \alpha .
\end{aligned}
$$

The second implication easily shows $y=\lambda \bar{x}$ for some number $\lambda>0$. Since $0 \in N_{F}(\bar{x})$, the first implication shows $\alpha \geq 0$, and consequently, by positive homogeneity,

$$
c \in N_{F}(\bar{x}) \Rightarrow\langle c, y\rangle \leq 0
$$


and consequently

$$
c \in N_{F}(\bar{x}) \Rightarrow\langle c, \bar{x}\rangle \leq 0 .
$$

But since $\bar{x}$ lies on the boundary of the set $F$, there exists a nonzero vector $\bar{c} \in N_{F}(\bar{x})$, and since zero lies in the interior of $F$, there exists a number $\delta>0$ such that $\delta \bar{c} \in F$. Hence

$$
0 \geq\langle\bar{c}, \delta \bar{c}-\bar{x}\rangle>-\langle\bar{c}, \bar{x}\rangle
$$

which is a contradiction.

Corollary 4. Suppose the compact convex set $F \subset \mathbf{R}^{n}$ contains zero in its interior. Then a vector $c$ lies in the relative interior of a proper exposed face of the polar set $F^{\circ}$ if and only if the problem $\max _{F}\langle c, \cdot\rangle$ has a nondegenerate maximizer with optimal value $\sigma_{F}(c)=1$.

Proof. By the preceding proposition, there exists a point $\bar{x}$ on the boundary of $F$ such that $c \in \operatorname{ri} N_{F}(\bar{x})$ and $\langle c, \bar{x}\rangle=1$. This point $\bar{x}$ is the desired nondegenerate maximizer, and clearly $\sigma_{F}(c)=\langle c, \bar{x}\rangle=1$.

Conversely, if $\bar{x}$ is a nondegenerate maximizer and $\sigma_{F}(c)=1$, then by definition $c \in \operatorname{ri} N_{F}(\bar{x})$ and $\langle c, \bar{x}\rangle=1$. Clearly $c \neq 0$, so $\bar{x}$ must lie on the boundary of $F$, and the result now follows by the preceding proposition.

Theorem 5 (Generic nondegeneracy). For any nonempty compact convex set $F \subset \mathbf{R}^{n}$, the set of vectors $c \in \mathbf{R}^{n}$ with the property that the problem $\max _{F}\langle c, \cdot\rangle$ has no nondegenerate maximizers has measure zero.

Proof. To prove this result, we use the idea of Hausdorff measure, for which a good basic reference is [35]. We consider the subset $H$ of the unit sphere consisting of vectors $c$ such that the linear function $\langle c, \cdot\rangle$ has no nondegenerate maximizers over the set $F$. The unit sphere has dimension $n-1$; we show that $H$ has $(n-1)$-dimensional Hausdorff measure zero. It follows easily that the cone $\mathbf{R}_{+} H$ has measure zero, which is the result we desire.

We first restrict attention to the case when $F$ contains zero in its interior. The general case follows easily, first by a translation to ensure zero lies in ri $F$ and then by considering $\mathbf{R}^{n}$ as the direct sum of the span of $F$ and its orthogonal complement.

Assume henceforth, therefore, that the compact convex set $F$ contains zero in its interior. Its polar $F^{\circ}$ is then another compact convex set containing zero in its interior. Consider the following map from the boundary of $F^{\circ}$ to 
the unit sphere $S^{n-1}$. We define $\Phi: \operatorname{bd}\left(F^{\circ}\right) \rightarrow S^{n-1}$ by $\Phi(x)=|x|^{-1} x$. Since the function $x \mapsto|x|^{-1}$ is locally Lipschitz on $\mathbf{R}^{n} \backslash\{0\}$, it is globally Lipschitz on the compact subset $\mathrm{bd}\left(F^{\circ}\right)$, and hence so is $\Phi$. Furthermore, $\Phi$ is invertible, with inverse $\Phi^{-1}: S^{n-1} \rightarrow \mathrm{bd}\left(F^{\circ}\right)$ given by $\Phi^{-1}(x)=\left(\gamma_{F^{\circ}}(x)\right)^{-1} x$. The sublinear function $\gamma_{F^{\circ}}=\sigma_{F}$ is everywhere finite, and hence Lipschitz, so the function $x \mapsto\left(\gamma_{F^{\circ}}(x)\right)^{-1}$ is locally Lipschitz on $\mathbf{R}^{n} \backslash\{0\}$, and hence globally Lipschitz on $S^{n-1}$, and therefore so is $\Phi^{-1}$. Thus $\Phi$ is a Lipschitz homeomorphism between the compact sets $\operatorname{bd}\left(F^{\circ}\right)$ and $S^{n-1}$, with Lipschitz inverse. Consequently, it is easy to see that $\Phi$ and $\Phi^{-1}$ preserve sets of Hausdorff measure zero. It therefore suffices to prove the result with $\operatorname{bd}\left(F^{\circ}\right)$ in place of $S^{n-1}$.

Consider, therefore, any vector $c \in \operatorname{bd}\left(F^{\circ}\right)$ such that the optimization problem $\max _{F}\langle c, \cdot\rangle$ has no nondegenerate maximizers. The intersection of $F^{\circ}$ with a supporting hyperplane at $c$ gives a proper exposed face $G$ of $F$ containing $c$. Furthermore, Corollary 4 implies $c \notin \mathrm{ri} G$, so $c$ must lie in the relative boundary of $G$. The result now follows, since by a result of Larman [17], the union of the relative boundaries of the proper faces of an $n$ dimensional compact convex set has $(n-1)$-dimensional Hausdorff measure zero.

Corollary 6 (Generic nondegenerate maximization and quadratic decay). Denote by $L$ the set of vectors $c \in \mathbf{R}^{n}$ such that the linear functional $\langle c, \cdot\rangle$ decays quadratically around a nondegenerate maximizer over the set $F$. Then the cone $L \cup\{0\}$ is full measure in $\mathbf{R}^{n}$.

Proof. This result follows by combining Theorem 5 with Proposition 2.

It is interesting to compare this approach to nondegeneracy with the development of Pataki and Tunçel [29]. Their framework consists of a primal conic convex program,

$$
\inf _{(L+b) \cap K}\langle d, \cdot\rangle,
$$

where $L$ is a linear subspace and $K$ is a pointed closed convex cone with nonempty interior, and a corresponding dual problem

$$
\inf _{\left(L^{\perp}+d\right) \cap K^{+}}\langle b, \cdot\rangle,
$$

where $K^{+}$is the dual cone $\{s:\langle z, s\rangle \geq 0 \forall z \in K\}$. For simplicity, suppose $0 \in \operatorname{int} F$. Then by choosing

$$
d=(-c, 0) \in \mathbf{R}^{n} \times \mathbf{R}, \quad L=\mathbf{R}^{n} \times\{0\}, \quad b=(0,1), \quad K=\mathbf{R}_{+}(F \times\{1\}),
$$


we arrive at a primal conic convex program equivalent to $\max _{F}\langle c, \cdot\rangle$. The dual problem is easy to construct (since $K^{+}=\mathbf{R}_{+}\left(-F^{\circ} \times\{1\}\right)$ ), and reduces to $\inf \left\{r:(c, r) \in\right.$ epi $\left.\sigma_{F}\right\}$, so the dual optimal solution is $-\left(c, \sigma_{F}(c)\right)$.

Now suppose the problem $\max _{F}\langle c, \cdot\rangle$ has unique solution $x_{c}$. Then the unique optimal solution of the corresponding primal conic convex program is $\left(x_{c}, 1\right)$, which lies in the relative interior of the exposed face $G=\mathbf{R}_{+}\left(x_{c}, 1\right)$. In the language of [29], strict complementarity in this case amounts to the optimal dual solution lying in the relative interior of the conjugate face, which reduces to

$$
G^{\triangle}=\left\{\left(-y,\left\langle x_{c}, y\right\rangle\right): y \in N_{F}\left(x_{c}\right)\right\}
$$

(via calculations similar to those above). Strict complementarity therefore holds exactly when $c \in$ ri $N_{F}\left(x_{c}\right)$, the property we refer to as "nondegeneracy". While [29] shows that strict complementarity is a generic property, that result concerns variations to the whole data triple $(d, b, L)$, so is independent of ours.

\section{Semi-algebraic functions and stratification}

When our underlying feasible region $F$ is semi-algebraic, the rather classical arguments we presented in the previous section have much stronger implications. We therefore next introduce the semi-algebraic tools we use, and discuss their immediate implications.

As we remarked in the introduction, a subset of $\mathbf{R}^{n}$ is semi-algebraic if it is a finite union of sets, each defined by finitely-many polynomial inequalities. A function (or set-valued mapping) is semi-algebraic if its graph is semialgebraic.

Semi-algebraic sets and functions enjoy many structural properties. In particular, every semi-algebraic set can be written as a finite disjoint union of manifolds (or "strata") that fit together in a regular "stratification": see [2], for example, or the exposition in $[42, \S 4.2]$. In particular, the dimension of a semi-algebraic set is the maximum of the dimensions of the strata, a number independent of the stratification: see [8, Definition 9.14] for more details.

This paper concerns "generic" properties. It is therefore worth emphasizing that, as an immediate consequence of stratification, the following four properties of semi-algebraic sets $S \subset \mathbf{R}^{n}$ are equivalent: 
- $S$ is dense;

- the dimension of the complement of $S$ is strictly less than $n$;

- $S$ is full-measure;

- $S$ is topologically generic.

To see this, note that the complement $S^{c}$ is semi-algebraic, and hence is a finite union of (relatively open) manifolds $M_{j}$, and by definition, $\operatorname{dim} S^{c}<n$ if and only if $\operatorname{dim} M_{j}<n$ for all $j$. If $S^{c}$ is dense, then none of the manifolds $M_{j}$ can be open, and hence $\operatorname{dim} M_{j}<n$ for all $j$. Conversely, if $\operatorname{dim} M_{j}<n$ for all $j$, then each complement $M_{j}^{c}$ is both full-measure and topologically generic, whence so is their intersection, namely $S$. Since, in general, full-measure or topologically generic sets are always dense, the equivalence follows.

In this paper we make fundamental use of a stratification result. We present a particular case - adapted to our needs - of a more general result: see $[42$, p. $502, \S 1.19(2)]$ or $[24]$ for the statement in its full generality. The result describes a decomposition of the domain of a semi-algebraic function into subdomains on which the function has "constant rank": a smooth function has constant rank if its derivative has constant rank throughout its domain. Such functions have a simple canonical form: they are locally equivalent to projections, as described by the following result from basic differential geometry [18, Thm 7.8].

Proposition 7 (Constant Rank Theorem). Let $M_{1}$ and $M_{2}$ be two differentiable manifolds, of dimensions $m_{1}$ and $m_{2}$ respectively, and let $g: M_{1} \rightarrow M_{2}$ be a differentiable mapping of constant rank $r \leq \min \left\{m_{1}, m_{2}\right\}$. Then for every point $x \in M_{1}$, there exist neighborhoods $O_{i}$ of zero in $\mathbf{R}^{m_{i}}$ and local diffeomorphisms $\psi_{i}: O_{i} \rightarrow M_{i}$ (for $\left.i=1,2\right)$ with $\psi_{1}(0)=x$ and $\psi_{2}(0)=g(x)$, such that mapping $\psi_{2}^{-1} \circ \mathrm{g} \circ \psi_{1}$ is just the projection $\pi: O_{1} \rightarrow O_{2}$ defined by

$$
\pi\left(y_{1}, y_{2}, \ldots, y_{m_{1}}\right)=\left(y_{1}, y_{2}, \ldots, y_{r}, 0, \ldots, 0\right) \in \mathbf{R}^{m_{2}}, \quad\left(y \in O_{1}\right) .
$$

The key stratification result we use follows. For our purposes in this work, readers unfamiliar with differential geometry could simply replace the term "stratification" by "decomposition into a disjoint union of subsets".

Proposition 8 (Map stratification). Let $f: M \rightarrow \mathbf{R}^{m}$ be a semi-algebraic function, where $M$ is a semi-algebraic subset of $\mathbf{R}^{n}$. Then, for any integer 
$k=1,2, \ldots$, there exist a stratification $\mathcal{S}=\left\{S_{i}\right\}_{i}$ of $M$ into $C^{k}$ semi-algebraic submanifolds, and a stratification $\mathcal{T}$ of $\mathbf{R}^{m}$ into $C^{k}$ semi-algebraic submanifolds, such that the restriction $f_{i}$ of $f$ onto each stratum $S_{i} \in \mathcal{S}$ is a $C^{k}$ semi-algebraic function, $f_{i}\left(S_{i}\right) \in \mathcal{T}$, and $f_{i}$ is of constant rank in $S_{i}$.

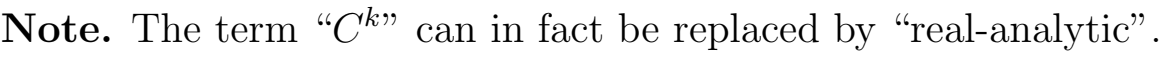

The above proposition yields that each restriction $f_{i}: S_{i} \rightarrow f_{i}\left(S_{i}\right)$ is surjective, $C^{k}$, and of constant rank $r_{i}$. Thus $r_{i}$ is also equal to the dimension of the manifold $f_{i}\left(S_{i}\right)$ :

$$
r_{i}=\operatorname{rank} f_{i}=\operatorname{dim} \operatorname{Im}\left(d f_{i}(x)\right)=\operatorname{dim}\left(T_{f_{i}\left(S_{i}\right)}\right)\left(f_{i}(x)\right), \quad \text { for all } x \in S_{i} .
$$

Semi-algebraic assumptions strengthen conclusions about sensitivity analysis, as the following refinement of Corollary 6 shows.

Corollary 9 (Generic stability). For any nonempty semi-algebraic compact convex set $F \subset \mathbf{R}^{n}$, and any integer $k=1,2, \ldots$, there exists a dense open semi-algebraic set $G \subset \mathbf{R}^{n}$ with the following property. The semi-algebraic map $c \mapsto x_{c}$, taking vectors $c \in \mathbf{R}^{n}$ to the minimum-norm solution of the optimization problem $\max _{F}\langle c, \cdot\rangle$, is $C^{k}$-smooth throughout $G$. Furthermore, for all vectors $c \in G$, the objective $\langle c, \cdot\rangle$ decays quadratically over $F$ around the optimal solution $x_{c}$, and $c \in$ ri $N_{F}\left(x_{c}\right)$.

Proof. The fact that the map $c \mapsto x_{c}$ is semi-algebraic follows by a standard argument using the Tarski-Seidenberg Theorem $[2,8]$. The existence of a dense open semi-algebraic set $G^{\prime} \subset \mathbf{R}^{n}$ on which this map is $C^{k}$ follows by applying the stratification result. Consider the full-measure set $L$ guaranteed by Corollary 6 . Since the set $F$ is semi-algebraic, another application of the Tarski-Seidenberg Theorem shows that so is the set $L$, and hence it must contain a dense open semi-algebraic subset, again using the stratification result. The result follows by defining $G=G^{\prime} \cap L$.

We can replace the term " $C^{k}$ " with "real-analytic".

\section{Partial smoothness}

The generic stability result, Corollary 9, does not yet capture the full force of classical sensitivity analysis under the second-order sufficient conditions, 
because it lacks the active set philosophy. We turn next, therefore, to the idea of partial smoothness introduced in [21], specialized to the case of a convex set $F$. This idea involves a continuity property of the normal cone mapping $N_{F}$, so we first recall the definition of continuity for a set-valued mapping.

In general, for two metric spaces $X$ and $Y$ and a set-valued mapping $T: X \rightrightarrows Y$, we say (cf. $[30,34]$ ) that $T$ is outer semicontinuous at a point $\bar{x} \in X$ if, for any sequence of points $x^{r} \in X$ converging to $\bar{x}$ and any sequence of points $y^{r} \in T\left(x^{r}\right)$ converging to $\bar{y}$, we must have $\bar{y} \in T(\bar{x})$. On the other hand, we say that $T$ is inner semicontinuous at $\bar{x}$ if, for any sequence of points $x^{r} \in X$ converging to $\bar{x}$ and any point $\bar{y} \in T(\bar{x})$, there exists a sequence $y^{r} \in Y$ converging to $\bar{y}$ such that $y^{r} \in T\left(x^{r}\right)$ for all large $r$. If both properties hold, we call $T$ continuous at $\bar{x}$.

Definition 10. A closed convex set $F \subset \mathbf{R}^{n}$ is called partly smooth at a point $\bar{x} \in F$ relative to a set $\mathcal{M} \subset F$ if the following properties hold:

(i) $\mathcal{M}$ is a $C^{2}$ manifold around $\bar{x}$ (called the active manifold).

(ii) The normal cone mapping $x \mapsto N_{F}(x)$, restricted to the domain $\mathcal{M}$, is continuous at $\bar{x}$.

(iii) $N_{\mathcal{M}}(\bar{x})=N_{F}(\bar{x})-N_{F}(\bar{x})$.

Some comments are in order about this definition, which, in this convex case, was shown in [21] to be exactly equivalent to the definition of an identifiable surface introduced by Wright [43].

The idea of a manifold that we use here is rudimentary. Following [34], we say that a set $\mathcal{M} \subset \mathbf{R}^{n}$ is a $C^{2}$ manifold (of codimension $m$ ) around a point $\bar{x} \in \mathcal{M}$ if there exists a $C^{2}$ map $G: \mathbf{R}^{n} \rightarrow \mathbf{R}^{m}$ with the properties that $G(\bar{x})=0$, the derivative map $d G(\bar{x}): \mathbf{R}^{n} \rightarrow \mathbf{R}^{m}$ is surjective and the inverse image $G^{-1}(0)$ coincides with $\mathcal{M}$ on a neighborhood of $\bar{x}$ : we refer to $G(x)=0$ as a local equation for $\mathcal{M}$. We remark that, in the standard language of differential geometry, $C^{2}$ submanifolds of $\mathbf{R}^{n}$ have this property around every point.

The definition of partial smoothness involves several interpretations of the normal cone. Given a point $x \in F$, the set $N_{F}(x)$ is the usual normal cone, in the sense of convex analysis [32]. We can regard $N_{F}$ as a set-valued mapping $x \mapsto N_{F}(x)$. On the other hand, $N_{\mathcal{M}}(\bar{x})$ is the normal space in the usual sense of differential geometry: the orthogonal complement of the 
tangent space $T_{\mathcal{M}}(\bar{x})$. Given a representation as above of the set $\mathcal{M}$ as $G^{-1}(0)$ (locally), $T_{\mathcal{M}}(\bar{x})$ is just the null space of the derivative $d G(\bar{x})$ (independent of the choice of the map $G)$. The definition of both $N_{F}(\bar{x})$ and $N_{\mathcal{M}}(\bar{x})$ are subsumed by the more general variational-analytic idea of the normal cone developed in [34, 27].

Geometrically, condition (iii) guarantees that the set $F$ has a "sharpness" property around the active manifold $\mathcal{M}$, as illustrated in the following simple examples.

Example 1 (sharpness). In the space $\mathbf{R}^{3}$, define sets

$$
\begin{aligned}
& F_{1}=\left\{(u, v, w): w \geq u^{2}+|v|\right\} \\
& F_{2}=\left\{(u, v, w): w \geq(|u|+|v|)^{2}\right\} \\
& \mathcal{M}=\left\{\left(t, 0, t^{2}\right): t \in(-1,1)\right\} .
\end{aligned}
$$

Then the closed convex set $F_{1}$ is partly smooth at the point $\bar{x}=(0,0,0)$ relative to the active manifold $\mathcal{M}$. On the other hand, the closed convex set $F_{2}$ is not: an easy calculation shows

$$
\begin{aligned}
& N_{F_{2}}(0,0,0)=\{(0,0, w): w \leq 0\} \\
& N_{\mathcal{M}}(0,0,0)=\{(0, v, w): v, w \in \mathbf{R}\}
\end{aligned}
$$

so condition (iii) fails.

It is standard and easy to check that the set-valued mapping $x \mapsto N_{F}(x)$ is always outer semicontinuous on the set $F$, and hence on $\mathcal{M}$. Part (ii) of the definition of partial smoothness therefore reduces to the inner semicontinuity property. The following example illustrates how this continuity property can fail.

Example 2 (failure of normal continuity). In the space $\mathbf{R}^{3}$, define sets

$$
\begin{aligned}
F & =\left\{(u, v, w): v \geq 0, w \geq 0, v+w \geq u^{2}\right\} \\
\mathcal{M} & =\left\{\left(t, t^{2}, 0\right): t \in(-1,1)\right\} .
\end{aligned}
$$

The set $F$ is closed and convex, and conditions (i) and (iii) are satisfied at the point $\bar{x}=(0,0,0)$. However, condition (ii) fails, since the normal cone mapping is discontinuous at zero, relative to $\mathcal{M}$.

While not obvious from the above definition, the active manifold for a partly smooth convex set is locally unique around the point of interest: see [13, Cor. 4.2]. 
For the purposes of sensitivity analysis, partial smoothness is most useful when combined with a second-order sufficiency condition, captured by the following definition.

Definition 11. Consider a vector $\bar{c} \in \mathbf{R}^{n}$ and a closed convex set $F \subset \mathbf{R}^{n}$ that is partly smooth at a point $\bar{x} \in \operatorname{argmax}_{F}\langle\bar{c}, \cdot\rangle$ relative to a set $\mathcal{M} \subset F$. We say that $\bar{x}$ is strongly critical if the following properties hold:

(i) Nondegeneracy: $\bar{c} \in \operatorname{ri} N_{F}(\bar{x})$.

(ii) Quadratic decay: There exists a constant $\delta>0$ such that

$$
\langle\bar{c}, \bar{x}\rangle \geq\langle\bar{c}, x\rangle+\delta|x-\bar{x}|^{2} \quad \text { for all } x \in \mathcal{M} \text { sufficiently near } \bar{x} \text {. }
$$

The nondegeneracy condition is analogous to the "strict complementarity" condition in classical nonlinear programming. The classical tangent cone $T_{F}(\bar{x})=\operatorname{cl} \mathbf{R}_{+}(F-\bar{x})$ and the normal cone $N_{F}(\bar{x})$ are mutually dual, so

$$
\bar{c} \in N_{F}(\bar{x}) \Leftrightarrow\langle\bar{c}, d\rangle \leq 0 \text { for all } d \in T_{F}(\bar{x}),
$$

whereas [32, Thm 13.1] shows

$$
\bar{c} \in \operatorname{ri} N_{F}(\bar{x}) \Leftrightarrow\langle\bar{c}, d\rangle<0 \text { for all } d \in T_{F}(\bar{x}) \backslash\left(-T_{F}(\bar{x})\right) .
$$

Just as in the nonlinear programming case, assumptions (i) and (ii) yield uniqueness of the maximizer $\bar{x}$ for the optimization problem $\max _{F}\langle\bar{c}, \cdot\rangle$. The following analogue of classical sensitivity results shows that strong criticality also implies that good sensitivity properties hold, and that the active manifold, locally, is simply the set of optimal solutions of perturbed problems.

Theorem 12 (second-order sufficiency). Consider a vector $\bar{c} \in \mathbf{R}^{n}$ and a compact convex set $F \subset \mathbf{R}^{n}$ that is partly smooth at a point $\bar{x} \in \operatorname{argmax}_{F}\langle\bar{c}, \cdot\rangle$ relative to a set $\mathcal{M} \subset F$. Assume $\bar{x}$ is strongly critical. Then $\langle\bar{c}, \cdot\rangle$ is maximized over $F$ by $\bar{x}$, and decays quadratically around it. Furthermore, there exists a neighborhood $U$ of $\bar{c}$ and a $C^{1}$ map from $U$ to $\mathcal{M}$, denoted $c \mapsto x_{c}$, mapping a vector $c \in U$ to the unique optimal solution $x_{c}$ of the perturbed problem $\max _{F}\langle c, \cdot\rangle$. The set $\left\{x_{c}: c \in U\right\}$ is locally identical to $\mathcal{M}$ near $\bar{x}$.

With the exception of the last statement, this result can be found in [21] and [13, Thm 6.2]. The last statement amounts to the observation that every point in $\mathcal{M}$ near $\bar{x}$ can be written as $x_{c}$ for some vector $c \in U$. Consider any 
sequence $x^{r} \in \mathcal{M}$ approaching $\bar{x}$ for which this representation fails. Since the normal cone mapping $N_{F}$ is continuous when restricted to $\mathcal{M}$, there exist vectors $c^{r} \in N_{F}\left(x^{r}\right)$ approaching $\bar{c}$. Since $c^{r} \in U$ for all large indices $r$, we deduce $x_{r}=x_{c_{r}}$, which is a contradiction.

Just as in classical sensitivity analysis, the assumptions and conclusions of this theorem can fail, even on straightforward examples. A good illustration is the following example.

Example 3. Consider the convex optimization problem over $\mathbf{R}^{3}$,

$$
\inf \left\{w: w \geq(|u|+|v|)^{2}\right\} .
$$

As we perturb the linear objective function slightly, the corresponding optimal solution does not vary smoothly: it may lie on not one but two distinct manifolds containing zero. More precisely, consider the perturbed problem

$$
\inf \left\{-2 a u-2 b v+w: w \geq(|u|+|v|)^{2}\right\},
$$

for parameters $a, b \in \mathbf{R}$. When $|a|=|b| \neq 0$, the optimal solution is not unique. Furthermore, whenever $|a| \neq|b|$, the optimal solution is unique, but is given by

$$
\left\{\begin{array}{cc}
\left(a, 0, a^{2}\right) & (|a|>|b|) \\
\left(0, b, b^{2}\right) & (|a|<|b|) .
\end{array}\right.
$$

Clearly this optimal solution is not a smooth function of the parameters.

Nonetheless, following Spingarn and Rockafellar [40], we argue that, for a broad class of concrete optimization problems, such breakdowns are rare, and therefore that partial smoothness and strong criticality are reasonable assumptions. Specifically, we prove in the next section that, for nonempty semi-algebraic compact convex sets $F$, the second-order sufficiency assumptions of Theorem 12 hold generically.

\section{Main result}

Henceforth we assume that the nonempty compact convex set $F \subset \mathbf{R}^{n}$ is semi-algebraic. Our main result asserts that a generic linear optimization problem over $F$ has a unique optimal solution, that $F$ is partly smooth there, and strong criticality holds. The proof we develop shows how the corresponding active manifold arises naturally, by means of Proposition 8 (constant rank stratification) applied to an appropriate function. 
Theorem 13 (Generic partial smoothness). Given any nonempty compact convex semi-algebraic set $F \subset \mathbf{R}^{n}$, for almost all linear objective functions $\langle c, \cdot\rangle$ (indeed for all nonzero vectors $c$ in a dense open semi-algebraic cone in $\left.\mathbf{R}^{n}\right)$ the optimization problem $\max _{F}\langle c, \cdot\rangle$ has the following properties.

(i) Existence of a nondegenerate maximizer with quadratic decay, $x_{c} \in F$.

(ii) Partial smoothness of $F$ at $x_{c}$ relative to a semi-algebraic $C^{2}$ manifold $\mathcal{M}_{c} \subset F$.

(iii) Local uniqueness of the active manifold $\mathcal{M}_{c}$ near $x_{c}$, and "local constancy": there exists a semi-algebraic $C^{2}$ manifold $\mathcal{M}$ such that, for all vectors $c^{\prime}$ sufficiently near $c$, we can choose $\mathcal{M}_{c^{\prime}}=\mathcal{M}$.

(iv) $C^{1}$-smooth dependence of the unique optimal solution of the perturbed problem $\max _{F}\left\langle c^{\prime}, \cdot\right\rangle$ as the vector $c^{\prime}$ varies near $c$ : furthermore, this optimal solution lies on the active manifold $\mathcal{M}$.

Notes: Before we begin the proof, we make some comments about this result. As we have observed, any dense semi-algebraic subset of $\mathbf{R}^{n}$ must be full-measure and topologically generic, with a complement whose dimension is strictly less than $n$, and it must contain a dense open semi-algebraic subset. By an obvious positive homogeneity argument, it suffices to find a dense semi-algebraic subset of the unit sphere $S^{n-1}$ on which the conclusions of the theorem hold.

It is interesting to revisit the simple convex optimization problem (6). Truncating the feasible region (by intersecting with the unit ball for example), we obtain a convex compact semi-algebraic set over which the functional $\bar{c}=(0,0,-1)$ has a unique maximizer (the origin) satisfying the first generic condition asserted in the theorem, while failing the last three. Thus the vector $\bar{c}$ lies outside the asserted dense semi-algebraic set.

Proof. Let us consider the set-valued mapping $\tilde{\Phi}: S^{n-1} \rightrightarrows F$ defined by

$$
\tilde{\Phi}(c)=\operatorname{argmax}_{F}\langle c, \cdot\rangle,
$$

so in fact $\tilde{\Phi}=\left.\left(\partial \sigma_{F}\right)\right|_{S^{n-1}}$ and

$$
\tilde{\Phi}^{-1}(x)=N_{F}(x) \cap S^{n-1} .
$$


The Tarski-Seidenberg Theorem $[2,8]$ shows that $\tilde{\Phi}$ is semi-algebraic. Let $L$ denote the subset of $\mathbf{R}^{n}$ asserted in Corollary 6. Since $L \cup\{0\}$ is a dense cone, the set $D=L \cap S^{n-1}$ is dense in the sphere $S^{n-1}$. Since the set $F$ is semialgebraic, so is $D$, again by the Tarski-Seidenberg Theorem. Consequently, the set

$$
N_{*}=S^{n-1} \backslash D
$$

has dimension strictly less than $n-1$.

Let $\Phi: D \rightarrow F$ denote the restriction of the mapping $\tilde{\Phi}$ to $D$, so in other words $\Phi=\left.\left(\partial \sigma_{F}\right)\right|_{D}$. Observe that $\Phi$ is single-valued: in our previous notation, for all vectors $c \in D$ we have $\Phi(c)=x_{c}$ and the strict complementarity and quadratic decay conditions hold:

(i) $c \in \operatorname{ri} N_{F}(\Phi(c))$;

(ii) for some $\delta>0$,

$$
\langle c, \Phi(c)\rangle \geq\langle c, x\rangle+\delta|x-\Phi(c)|^{2} \quad \text { for all } x \in F .
$$

Applying Proposition 8 (Map stratification) to the semi-algebraic function $\Phi$, we arrive at a stratification $\mathcal{S}=\left\{S_{j}\right\}_{j \in J}$ of $D$ such that for every index $j \in J$,

- $\Phi_{j}:=\left.\Phi\right|_{S_{j}}$ is a $C^{2}$ function of constant rank;

- $\Phi_{j}\left(S_{j}\right)$ is a manifold of dimension equal to the rank of $\Phi_{j}$;

- the image strata $\left\{\Phi\left(S_{j}\right)\right\}_{j}$ belong to a stratification of $\mathbf{R}^{n}$.

In particular,

$$
D=\bigcup_{j \in J} S_{j}
$$

and

$$
j_{1} \neq j_{2} \quad \Rightarrow \quad \Phi\left(S_{j_{1}}\right)=\Phi\left(S_{j_{2}}\right) \quad \text { or } \quad \Phi\left(S_{j_{1}}\right) \cap \Phi\left(S_{j_{2}}\right)=\emptyset .
$$

Denote the set of strata of full dimension by $\left\{S_{j_{1}}, \ldots, S_{j_{l}}\right\}$. Observe that the set

$$
U=\bigcup_{i=1}^{\ell} S_{j_{i}}
$$


is dense in $D$, and hence in $S^{n-1}$.

Our immediate objective is to show that for every vector $c \in U$, the set $F$ is partly smooth at $\Phi(c)$ with respect to some set $\mathcal{M} \subset F$. To this end, fix any point $\bar{x} \in \Phi(U)$. For any point $x \in \Phi(U)$, define the set of "active" indices

$$
I(x):=\left\{j \in J: x \in \Phi\left(S_{j}\right)\right\} .
$$

We aim to show that the set $F$ is partly smooth at $\bar{x}$ relative to the set

$$
\mathcal{M}=\Phi_{j}\left(S_{j}\right)
$$

for any index $j \in I(\bar{x})$. Note that, in view of property (11), the definition of $\mathcal{M}$ is in fact independent of the choice of index $j$, and for the same reason the set of active indices $I(x)$ is independent of the point $x \in \mathcal{M}$. In what follows, we simply write $I$ for the set $I(\bar{x})$.

Clearly, property (i) of the definition of partial smoothness (Definition 10) holds. If we can prove properties (ii) and (iii), then our result will follow from Corollary 6 , since $U \subset D$.

\section{Step 1: normal cone continuity}

We establish inner continuity (and hence continuity) at $\bar{x}$ of the normal cone mapping $x \mapsto N_{F}(x)$ as $x$ moves along the manifold $\mathcal{M}$. It suffices to prove that the truncated normal cone mapping $\tilde{\Phi}^{-1}$ defined by equation (8) is inner semicontinuous. We proceed by decomposition with respect to the active strata.

For any point $x \in \mathcal{M}$, define

$$
N_{j}(x)=N_{F}(x) \cap S_{j} \quad(j \in J) .
$$

Notice

$$
N_{j}(x) \neq \emptyset \Leftrightarrow j \in I \Leftrightarrow \mathcal{M}=\Phi\left(S_{j}\right) .
$$

We therefore have

$$
N_{F}(x) \cap S^{n-1}=N_{*}(x) \cup \bigcup_{j \in I} N_{j}(x)
$$

where $N_{*}(x)=N_{F}(\bar{x}) \cap N_{*}$ for the set $N_{*}$ is defined by equation (9).

Claim A. For every point $x \in \mathcal{M}$ the set $\cup_{j \in I} N_{j}(x)$ is dense in $N_{F}(x) \cap S^{n-1}$. 
Proof of Claim A. Since we are assuming $\bar{x} \in \Phi(U)$, there exists an active index $j_{p}$ with $p \in\{1, \ldots, \ell\}$ corresponding to a full-dimensional stratum $S_{j_{p}}$ such that $\mathcal{M}=\Phi_{j_{p}}\left(S_{j_{p}}\right)$ (see property (13)). This yields that for every point $x \in \mathcal{M}$, there exists a vector $c \in S_{j_{p}}$ with $x=\Phi(c)$. Hence

$$
c \in N_{F}(x) \cap S_{j_{p}}=N_{j_{p}}(x) \subset \bigcup_{j \in I} N_{j}(x) .
$$

Now fix any vector $c_{*} \in N_{F}(x) \cap S^{n-1}$, and consider the spherical path

$$
c_{t}:=\frac{c+t\left(c_{*}-c\right)}{\left|c+t\left(c_{*}-c\right)\right|} \quad(t \in[0,1]) .
$$

Since $x=\Phi(c)$, we in fact know $c \in$ ri $N_{F}(x)$. It follows that $c_{t} \in \operatorname{ri} N_{F}(x)$, for all $t \in[0,1)$. Since $c \in S_{j_{p}} \subset D$, there exists a constant $\delta_{c}>0$ such that

$$
\langle c, x\rangle \geq\left\langle c, x^{\prime}\right\rangle+\delta_{c}\left|x-x^{\prime}\right|^{2} \quad \text { for all } x^{\prime} \in F .
$$

By the definition of the normal cone, we also have

$$
\left\langle c_{*}, x\right\rangle \geq\left\langle c_{*}, x^{\prime}\right\rangle \text { for all } x^{\prime} \in F
$$

Multiplying these inequalities by $(1-t)$ and $t$ respectively, and adding, we infer that the point $x$ is a maximizer of the function $\left\langle c_{t}, \cdot\right\rangle$ over the set $F$, with quadratic decay, for all $0 \leq t<1$. In other words, $c_{t} \in N_{F}(x) \cap D$, which in view of equation (10) yields $c_{t} \in \cup_{j \in I} N_{j}(x)$, for $t \in[0,1)$. Since $c_{t} \rightarrow c_{*}$ as $t \uparrow 1$, Claim A follows.

In view of Claim A, it is sufficient to establish the inner continuity of the mapping

$$
x \mapsto \bigcup_{j \in I} N_{j}(x) \quad x \in \mathcal{M} .
$$

To see this, we use the following simple and routine result: for completeness, we provide a proof.

Lemma 14. Let $X$ and $Y$ be metric spaces, and consider two set-valued mappings $G, T: X \rightrightarrows Y$ such that $\operatorname{cl}(G(x))=T(x)$ for all points $x \in X$. If $G$ is inner semicontinuous at a point $\bar{x} \in X$, then so is $T$. 
Proof of Lemma 14. Assume (towards a contradiction) that there exists a constant $\rho>0$, a sequence $\left\{x^{k}\right\} \subset X$ with $x^{k} \rightarrow \bar{x}$ and a point $\bar{y} \in T(\bar{x})$, such that

$$
\operatorname{dist}\left(\bar{y}, T\left(x^{k}\right)\right)>\rho>0 .
$$

Then pick any point $\hat{y} \in B(\bar{y}, \rho / 2) \cap G(\bar{x})$ and use the inner semicontinuity of $G$ to get a sequence of points $y^{k} \in G\left(x^{k}\right) \subset T\left(x^{k}\right)$ for $k \in \mathbf{N}$ such that $y^{k} \rightarrow \hat{y}$. This gives a contradiction, proving the lemma.

Applying this lemma to the set-valued mappings

$$
G(x)=\bigcup_{j \in I} N_{j}(x) \quad \text { and } \quad T(x)=N_{F}(x) \cap S^{n-1}
$$

accomplishes the reduction we seek.

Let us therefore prove the inner semicontinuity of the mapping defined in (14) at the point $\bar{x}$. To this end, fix any vector $\bar{c} \in \cup_{j \in I} N_{j}(\bar{x})$ and consider any sequence $\left\{x^{k}\right\} \subset \mathcal{M}$ approaching $\bar{x}$. For some index $j \in I$ we have $\bar{c} \in S_{j}$. Let us restrict our attention to the constant-rank surjective mapping $\Phi_{j}: S_{j} \rightarrow \mathcal{M}$ and let us recall that

$$
\Phi_{j}\left(S_{j}\right)=\mathcal{M} \quad \text { and } \quad \Phi_{j}(\bar{c})=\bar{x}
$$

Let $d$ be the dimension of the stratum $S_{j}$, so

$$
\operatorname{rank}\left(d \Phi_{j}\right)=\operatorname{dim} \mathcal{M}:=r \leq d \leq n-1
$$

Denote by $0_{d}$ (respectively $0_{r}$ ) the zero vector of the space $\mathbf{R}^{d}$ (respectively $\mathbf{R}^{r}$ ). Then applying the Constant Rank Theorem (Proposition 7), we infer that for some constants $\delta, \varepsilon>0$ there exist diffeomorphisms

$$
\psi_{1}: B\left(0_{d}, \delta\right) \rightarrow S_{j_{0}} \cap B(\bar{c}, \varepsilon) \quad \text { and } \quad \psi_{2}: B\left(0_{r}, \delta\right) \rightarrow \mathcal{M} \cap B(\bar{x}, \varepsilon)
$$

such that

$$
\psi_{1}\left(0_{d}\right)=\bar{c} \quad \text { and } \quad \psi_{2}\left(0_{r}\right)=\bar{x},
$$

and such that all vectors $y \in B\left(0_{d}, \delta\right)$ satisfy

$$
\left(\psi_{2}^{-1} \circ \Phi_{j} \circ \psi_{1}\right)(y)=\pi(y)
$$

where for $y=\left(y_{1}, \ldots, y_{d}\right) \in \mathbf{R}^{d}$ we have

$$
\pi\left(y_{1}, \ldots, y_{r}, y_{r+1} \ldots, y_{d}\right)=\left(y_{1}, \ldots, y_{r}\right) \in B\left(0_{r}, \delta\right) \subset \mathbf{R}^{r} .
$$


We may assume the sequence $\left\{x^{k}\right\}$ lies in $\mathcal{M} \cap B(\bar{x}, \varepsilon)$. Thus, in view of definition (15), for every integer $k \in \mathbf{N}$ there exists a vector $z^{k}=\left(z_{1}^{k}, \ldots, z_{r}^{k}\right) \in$ $B\left(0_{r}, \delta\right)$ with

$$
\psi_{2}\left(z^{k}\right)=x^{k}
$$

Note $z^{k} \rightarrow 0_{r}=\left(\psi_{2}\right)^{-1}(\bar{x})$. Define vectors

$$
y^{k}:=\left(z_{1}^{k}, \ldots, z_{r}^{k}, 0, . ., 0\right) \in \mathbf{R}^{d}
$$

for every $k \in \mathbf{N}$. Since $z^{k} \in B\left(0_{r}, \delta\right)$, we know $y^{k} \in B\left(0_{d}, \delta\right)$, and clearly

$$
y^{k} \rightarrow 0_{d}
$$

We now define vectors $c^{k}:=\psi_{1}\left(y^{k}\right)$ for each $k$. In view of definition (15) we see that $c^{k} \in S_{j} \cap B(\bar{c}, \varepsilon)$, and in view of properties (19) and (16),

$$
c^{k} \rightarrow \psi_{1}\left(0_{d}\right)=\bar{c} \text { as } k \rightarrow \infty
$$

To complete the proof of inner semicontinuity, it remains to show $c^{k} \in$ $N_{F}\left(x^{k}\right)$. Since $\Phi_{j}\left(c^{k}\right)=\Phi_{j}\left(\psi_{1}\left(y^{k}\right)\right)$ we infer by properties (17) and (19) that

$$
\psi_{2}^{-1}\left(\Phi_{j}\left(c^{k}\right)\right)=\left(\psi_{2}^{-1} \circ \Phi_{j} \circ \psi_{1}\right)\left(y^{k}\right)=\pi\left(y^{k}\right)=z^{k} .
$$

Using now the fact that $\psi_{2}$ is a diffeomorphism, we deduce from equation (18) that $\Phi_{j}\left(c^{k}\right)=\psi_{2}\left(z^{k}\right)=x^{k}$. Thus $c^{k} \in \Phi_{j}^{-1}\left(x^{k}\right) \subset N_{F}\left(x^{k}\right)$ which completes the proof of inner semicontinuity and hence of Step 1.

\section{Step 2: sharpness}

It remains to verify that condition (iii) of Definition 10, namely

$$
N_{\mathcal{M}}(\bar{x})=N_{F}(\bar{x})-N_{F}(\bar{x}),
$$

is also fulfilled.

To this end, as in the proof of Claim A, we can choose an index $j \in I$ corresponding to a stratum $S_{j}$ of full dimension $(n-1)$ such that $\mathcal{M}=\Phi_{j}\left(S_{j}\right)$. Recall that the semi-algebraic $C^{2}$-mapping $\Phi_{j}: S_{j} \rightarrow \mathcal{M}$ is surjective and has constant rank $r=\operatorname{dim} \mathcal{M}$, so $\operatorname{dim} N_{\mathcal{M}}(\bar{x})=n-r$. It follows directly from the inclusion $\mathcal{M} \subset F$ that $N_{F}(\bar{x}) \subset N_{\mathcal{M}}(\bar{x})$. Since the right-hand side is a subspace, we deduce

$$
N_{F}(\bar{x})-N_{F}(\bar{x}) \subset N_{\mathcal{M}}(\bar{x})
$$


Since $\Phi_{j}$ is surjective and of maximal rank, we deduce easily that $\Phi_{j}^{-1}(\bar{x})$ is a semi-algebraic submanifold of $S^{n-1}$ of dimension $(n-1)-r$, which, in view of definition (8) and equation (12) yields

$$
\operatorname{dim}\left(N_{F}(\bar{x}) \cap S^{n-1}\right) \geq \operatorname{dim} N_{j}(\bar{x}) \geq(n-1)-r .
$$

Thus $\operatorname{dim} N_{F}(\bar{x}) \geq n-r$, which, along with inclusion (21), yields equation (20), as required.

A simple argument extends the main result to unbounded feasible regions.

Corollary 15 (Unbounded feasible regions). Given any nonempty closed convex semi-algebraic set $F \subset \mathbf{R}^{n}$, for almost all vectors $c$ in the domain of the support function $\sigma_{F}$, the optimization problem $\max _{F}\langle c, \cdot\rangle$ has all the properties described in Theorem 13.

Proof. It suffices to prove the result for all compact subsets $G$ of the interior of the domain of $\sigma_{F}$. Since $\sigma_{F}$ is locally Lipschitz throughout the interior of its domain, it is globally Lipschitz on any such set $G$. Denoting the Lipschitz constant by $L$, we note for all vectors $c \in G$ the property

$$
\operatorname{argmax}_{F}\langle c, \cdot\rangle=\partial \sigma_{F}(c) \subset B(0, L) .
$$

Thus the original problem $\max _{F}\langle c, \cdot\rangle$ is equivalent to a problem with a compact feasible region: $\max _{F \cap B(0, L)}\langle c, \cdot\rangle$. Applying the main result to this latter problem completes the proof.

An assumption like semi-algebraicity (or, more generally, tameness) is crucial for results like those above. To see this, consider first any closed proper convex function $f$ on $\mathbf{R}$. For any point $x$ in the interior of the domain of $f$, the epigraph of $f$ is partly smooth at the point $(x, f(x))$ if and only if $f$ is either nondifferentiable at $x$ or $C^{2}$ around $x$.

Now consider any strictly increasing function $h:[0,1] \rightarrow \mathbf{R}_{+}$that is discontinuous on a dense set. Define functions $g, f:[0,1] \rightarrow \mathbf{R}$ by

$$
g(y)=\int_{0}^{y} h(t) d t \quad f(x)=\int_{0}^{x} g(y) d y
$$

Then $f$ is $C^{1}$ and convex on the interval $(0,1)$, but nowhere $C^{2}$. Finally, let $f$ take the value $+\infty$ outside the interval $[0,1]$. Then, as we have observed, 
the closed convex set $F=$ epi $f$ is not partly smooth at any point $(x, f(x))$ with $x \in(0,1)$.

However, for this set $F$ and any vector $c \in \mathbf{R}^{2}$ satisfying $-c_{1} / c_{2}=g(x)$ and $c_{2}<0$, it is easy to check that the unique maximizer for the problem $\max _{F}\langle c, \cdot\rangle$ is exactly the point $\{(x, f(x))\}$. Thus the conclusion of Corollary 15 fails whenever the ratio $-c_{1} / c_{2}$ lies in the interval $(g(0), g(1))$ and $c_{2}<0$. A straightforward extension of this idea gives an example of a compact convex set $F \subset \mathbf{R}^{2}$ over which the function $\langle c, \cdot\rangle$ has a unique maximizer for every nonzero vector $c \in \mathbf{R}^{2}$, but such that $F$ is never partly smooth around that maximizer.

Acknowledgment A major part of this work was accomplished during a research visit of the second and third authors at the University Pierre et Marie Curie (Paris 6), in May 2008. The authors thank Dmitriy Drusviyatskiy for the observation following Theorem 12, and Victor Vinnikov for the suggestion to include the final example.

\section{References}

[1] F. Alizadeh, J.-P.A. Haeberly, and M.L. Overton. Complementarity and nondegeneracy in semidefinite programming. Mathematical Programming, 77:111-128, 1997.

[2] S. Basu, R. Pollack, and M.-F. Roy. Algorithms in Real Algebraic Geometry. Springer, Berlin, 2003.

[3] A. Ben-Tal and A. Nemirovski. Lectures on Modern Convex Optimization: Analysis, Algorithms, and Engineering Applications. SIAM, Philadelphia, 2001.

[4] J.F. Bonnans and A. Shapiro. Perturbation Analysis of Optimization Problems. Springer, New York, 2000.

[5] J.V. Burke, A.S. Lewis, and M.L. Overton. Optimal stability and eigenvalue multiplicity. Foundations of Computational Mathematics, 1:205$225,2001$.

[6] J.V. Burke and J.J. Moré. On the identification of active constraints. SIAM Journal on Numerical Analysis, 25:1197-1211, 1988. 
[7] F.H. Clarke, Yu.S. Ledyaev, R.J. Stern, and P.R. Wolenski. Nonsmooth Analysis and Control Theory. Springer-Verlag, New York, 1998.

[8] M. Coste. An Introduction to o-minimal Geometry, 1999. RAAG Notes, 81 pages, Institut de Recherche Mathématiques de Rennes.

[9] A. Daniilidis, C. Sagastizábal, and M. Solodov. Identifying structure of nonsmooth convex functions by the bundle technique. SIAM Journal on Optimization, 20:820-840, 2009.

[10] A.L. Dontchev and T. Zolezzi. Well-Posed Optimization Problems. Springer-Verlag, Berlin, 1993.

[11] G. Ewald, D.G. Larman, and C.A. Rogers. The directions of the line segments and of the $r$-dimensional balls on the boundary of a convex body in Euclidean space. Mathematika, 17:1-20, 1970.

[12] M.C. Ferris. Finite termination of the proximal point algorithm. Mathematical Programming, 50:359-366, 1991.

[13] W.L. Hare and A.S. Lewis. Identifying active constraints via partial smoothness and prox-regularity. Journal of Convex Analysis, 11:251266, 2004.

[14] J.W. Helton and J. Nie. Semidefinite representation of convex sets. Mathematical Programming, 122:21-64, 2010.

[15] A.D. Ioffe. An invitation to tame optimization. SIAM Journal on Optimization, 19:1894-1917, 2009.

[16] A.D. Ioffe and R. Lucchetti. Typical convex program is very well posed. Mathematical Programming, 104:483-499, 2005.

[17] D.G. Larman. On a conjecture of Klee and Martin for convex bodies. Proceedings of the London Mathematical Society, 23:668-682, 1971. Corrigendum: Vol. 36, p. 86.

[18] J.M. Lee. Introduction to Smooth Manifolds. Springer, New York, 2003.

[19] C. Lemaréchal, F. Oustry, and C. Sagastizábal. The $\mathcal{U}$-Lagrangian of a convex function. Transactions of the American Mathematical Society, 352:711-729, 2000. 
[20] C. Lemaréchal and C. Sagastizábal. Practical aspects of the MoreauYosida regularization: theoretical preliminaries. SIAM Journal on $O p$ timization, 7:367-385, 1997.

[21] A.S. Lewis. Active sets, nonsmoothness and sensitivity. SIAM Journal on Optimization, 13:702-725, 2003.

[22] A.S. Lewis and S.J. Wright. A proximal method for composite minimization, 2008. arXiv:0812.0423v1.

[23] A.S. Lewis and S.J. Wright. Identifying activity. SIAM Journal on Optimization, 2010. To appear. arXiv:0901.2668v1.

[24] T.L. Loi. Thom stratifications for functions defined in o-minimal structures on $(\mathbb{R},+, \cdot)$. Comptes Rendus de l'Académie des Sciences de Paris, Série 1, 324:1391-1394, 1997.

[25] R. Mifflin and C. Sagastizábal. Proximal points are on the fast track. Journal of Convex Analysis, 9:563-579, 2002.

[26] R. Mifflin and C. Sagastizábal. A VU algorithm for convex minimization. Mathematical Programming, 104:583-608, 2005.

[27] B.S. Mordukhovich. Variational Analysis and Generalized Differentiation, I: Basic Theory; II: Applications. Springer, New York, 2006.

[28] J. Nocedal and S.J. Wright. Nonlinear Programming. Springer-Verlag, New York, 1997.

[29] G. Pataki and L. Tunçel. On the generic properties of convex optimization problems in conic form. Mathematical Programming, 89:449-457, 2001.

[30] R.R. Phelps. Convex Functions, Monotone Operators and Differentiability, volume 1364 of Lecture Notes in Mathematics. Springer-Verlag, New York, second edition, 1993.

[31] S.M. Robinson. Constraint nondegeneracy in variational analysis. Mathematics of Operations Research, 28:201-232, 2003.

[32] R.T. Rockafellar. Convex Analysis. Princeton University Press, Princeton, N.J., 1970. 
[33] R.T. Rockafellar. Monotone operators and the proximal point algorithm. SIAM Journal on Control and Optimization, 14:877-898, 1976.

[34] R.T. Rockafellar and R.J.-B. Wets. Variational Analysis. Springer, Berlin, 1998.

[35] C.A. Rogers. Hausdorff Measures. Cambridge University Press, Cambridge, U.K., 1970.

[36] R. Saigal and C. Simon. Generic properties of the complementarity problem. Mathematical Programming, 4:324-335, 1973.

[37] A. Shapiro. First and second order analysis of nonlinear semidefinite programs. Mathematical Programming, 77:301-320, 1997.

[38] A. Shapiro and M.K.H. Fan. On eigenvalue optimization. SIAM Journal on Optimization, 5:552-568, 1995.

[39] J.E. Spingarn. On optimality conditions for structured families of nonlinear programming problems. Mathematical Programming, 22:82-92, 1982 .

[40] J.E. Spingarn and R.T. Rockafellar. The generic nature of optimality conditions in nonlinear programming. Mathematics of Operations Research, 4:425-430, 1979.

[41] D. Sun. The strong second order sufficient condition and constraint nondegeneracy in nonlinear semidefinite programming and their consequences. Mathematics of Operations Research, 31:761-776, 2006.

[42] L. van den Dries and C. Miller. Geometric categories and o-minimal structures. Duke Mathematics Journal, 84:497-540, 1996.

[43] S.J. Wright. Identifiable surfaces in constrained optimization. SIAM Journal on Control and Optimization, 31:1063-1079, 1993. 